\section{DOES THE ADDITION OF A RADIAL ARTERY GRAFT IMPROVE SURVIVAL AFTER HIGHER RISK CORONARY SURGERY? A PROPENSITY-SCORE ANALYSIS}

doi:10.1136/heartjnl-2011-300198.8

${ }^{1} \mathrm{C}$ H Yap, ${ }^{2} \mathrm{P}$ A Hayward, ${ }^{2} \mathrm{~W}$ Y Shi, ${ }^{1} \mathrm{D}$ T Dinh, ${ }^{1} \mathrm{C}$ M Reid, ${ }^{3,4} \mathrm{G}$ C Shardey, ${ }^{3,4} \mathrm{~J}$ A Smith. ${ }^{1}$ Department of Epidemiology and Preventative Medicine, Monash University, Melbourne, UK; ${ }^{2}$ Department of Cardiac Surgery, Austin Hospital, University of Melbourne, Melbourne, UK; ${ }^{3}$ Department of Cardiothoracic Surgery and Surgery, Monash Medical Centre, Monash University, Melbourne, UK; ${ }^{4}$ Department of Surgery, Monash Medical Centre, Monash University, Melbourne, UK

Introduction The use of the radial artery as a second arterial graft during coronary surgery has become popular due to high patency, encouraging clinical outcomes and low harvest site complication rates. However it is not clear whether higher risk patients derive such benefits. We sought to assess this by examining outcomes in higher risk subgroups.

Methods A multicentre database was analysed. From 2001 to 2009, 11388 patients underwent isolated multivessel coronary surgery. We identified a higher risk subgroup ( $n=3149)$ according to emergent status, coronary instability, low ejection fraction, aortic counterpulsation or anticoagulant status. Among these, 2231 (71\%) received at least 1 radial artery graft in addition to a left internal thoracic artery (LITA). The remaining 918 (29\%) received LITA and veins only. Propensity-score matching and adjustment was performed to correct for group differences.

Results Patients who did not receive a radial artery were more likely to be older (mean age, radial: $66 \pm 10$ years vs vein: $71 \pm 10, p<0.0001$ ) female $(22 \%$ vs $27 \%, p=0.002)$, have poor left ventricular function ( $16 \%$ vs $23 \%$, $p<0.0001$ ), left main stenosis ( $35 \%$ vs $41 \%$, $p=0.002$ ) or be of emergent status ( $11 \%$ vs $24 \%, p<0.0001)$. These patients experienced higher unadjusted 30 -day mortality ( $2.2 \%$ vs $7.1 \%, p<0.0001)$ and poorer 7 -year survival $(\mathrm{p}<0.0001)$. Furthermore, 548 patients in the radial group were propensity-score matched to 548 receiving LITA and veins. At 30 days, there were comparable rates of mortality (radial: $2 \%$ vs vein: $3 \%, p=0.19)$, stroke ( $1 \%$ vs $1 \%, p=0.51)$, myocardial infarction ( $1 \%$ vs $1 \%, p=0.77)$, major adverse cardiac or cerebrovascular events (MACCE) ( $2 \%$ vs $4 \%, p=0.12)$, return to theatre ( $5 \%$ vs $7 \%, p=0.19)$, hospital readmissions $(12 \%$ vs $12 \%, p>0.99)$ and combined any mortality/morbidity ( $30 \%$ vs $32 \%, p=0.33$ ). At 7 years, survival between radial and vein groups was similar $(79 \pm 2.5 \%$ vs $80 \pm 2.5 \%, \mathrm{p}=0.74)$. Propensity-adjusted multivariable regression did not show radial artery to be protective from 30 -day mortality $(p=0.14$, OR $0.67,0.40$ to 1.13 ), 30-day MACCE ( $\mathrm{p}=0.23$, OR $0.76,0.48$ to 1.20 ), or mid-term mortality ( $\mathrm{p}=0.79$, HR $0.97,0.78$ to 1.20 ).

Conclusions This multicentre analysis suggests that patients with the greatest coronary instability, urgency of surgery, or impairment of ventricular function are not disadvantaged in the early and midterm by use of a single arterial graft. Limitations include the inability to correct for unquantifiable variables retrospectively. Despite this, surgeons may utilise clinical judgement to select radial or venous conduits to supplement the LITA according to other patient factors or technical preference without prejudicing outcome.

\section{EARLY HOSPITAL DISCHARGE AT 48 H FOLLOWING PRIMARY PCI FOR MYOCARDIAL INFARCTION IS BOTH SAFE AND FEASIBLE}

doi:10.1136/heartjnl-2011-300198.9

0 Guttmann, D A Jones, K S Rathod, M Akhtar, A Ludman, A K Jain, C Knight, A Mathur, S Mohiddin, A Wragg, E J Smith. Barts and the London NHS trust, London, $U K$

Introduction Reperfusion therapy with primary PCI (PPCI) has reduced rates of recurrent ischaemia and arrhythmia following ST elevation myocardial infarction (STEMI), resulting in shorter hospital stays. Discharge at $72 \mathrm{~h}$ in selected patients has been suggested. We investigated the feasibility and safety of very early discharge $(<48 \mathrm{~h})$ coupled with regular outpatient support for lowrisk patients following PPCI.

Methods 2317 patients underwent PPCI for STEMI between October 2003 and May 2010 at a regional Heart Attack Centre (HAC). Demographic and procedural data were documented at the time of intervention. Patients with TIMI 3 flow, ST segment resolution, good or moderate left ventricular function, and no dysrhythmia were stratified to $48 \mathrm{~h}$ discharge. Remaining patients were discharged according to physician preference. All patients were reviewed at 1,8 and 52 weeks with a multidisciplinary team including rehabilitation, heart failure, and psychology. The primary endpoint was major adverse cardiac events (MACE) included death, myocardial infarction (MI), stroke and target vessel revascularisation (TVR). All-cause mortality data were provided by the Office of National Statistics via the BCIS CCAD national audit. Outcomes were compared between those discharged at $\leq 48 \mathrm{~h}, 72 \mathrm{~h}$, and $>72 \mathrm{~h}$, out to 5 years of follow-up.

Results 1079 patients (46.5\%) were stratified to 48-h discharge, $14 \%$ discharged at $72 \mathrm{~h}$ and the remainder discharged at a median of 6 days (4.3-10), including those with complications. Patients discharged at $\leq 48 \mathrm{~h}$ were significantly younger and had a lower incidence of multi-vessel disease than those discharged at $72 \mathrm{~h}$ (Abstract 9 table 1). Remaining baseline characteristics were similar. MACE at 3 years was similar between 48 - $\mathrm{h}$ discharge patients and 72 - $h$ discharge $(9.1 \%$ vs $8.7 \%, p=0.7)$. This persisted out to 5 years $(9.6 \%$ vs $9 \%, p=0.55)$. As expected patients with length of stays $>72 \mathrm{~h}$ had significantly worse outcomes (Abstract 9 figure 1).

\section{Abstract 9 Table 1}

\begin{tabular}{lccc}
\hline & $\mathbf{4 8} \mathbf{h}(\mathbf{n}=\mathbf{1 0 7 9})$ & $\mathbf{7 2} \mathbf{h}(\mathbf{n}=\mathbf{3 2 3})$ & $\mathbf{p ~ V a l u e}$ \\
\hline Age & 60.7 & 64.0 & 0.0002 \\
Previous MI & $130(12.0 \%)$ & $35(10.8 \%)$ & 0.5569 \\
Previous CABG & $21(1.9 \%)$ & $7(2.2 \%)$ & 0.8019 \\
Previous PCI & $102(9.4 \%)$ & $29(9.0 \%)$ & 0.8007 \\
DM & $156(14.4 \%)$ & $52(16.1 \%)$ & 0.4632 \\
HTN & $455(42.1 \%)$ & $148(45.8 \%)$ & 0.2854 \\
Hchol & $403(37.3 \%)$ & $124(38.4 \%)$ & 0.7858 \\
3 vessel disease & $448(46.4 \%)$ & $156(54.9 \%)$ & 0.0112 \\
\hline
\end{tabular}

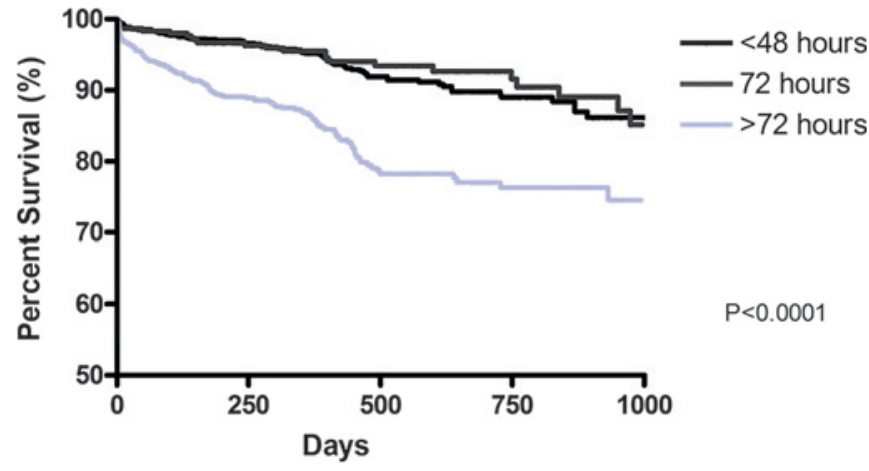

Abstract 9 Figure 1 MACE after primary PCl.

Conclusion Early discharge at $48 \mathrm{~h}$ is feasible and appears to be safe for patients undergoing contemporary Primary PCI. 